\title{
SCHARFRICHTER IN PREUSSISCHEN UND LIVLÄNDISCHEN STÄDTEN UM DIE WENDE DES MITTELALTERS UND DER FRÜHEN NEUZEIT
}

BEMERKUNGEN ZUM PROJEKT „INFAMIE (EHRLOSIGKEIT) IN PREUSSISCHEN, LIVLÄNDISCHEN UND NORDWESTRUSSISCHEN STÄDTEN IN MITTELALTER UND IN DER FRÜHEN NEUZEIT“"

von Paweł A. Jeziorski

\section{I}

Das Projekt „Infamie (Ehrlosigkeit) in preußischen, livländischen und nordwestrussischen Städten im Mittelalter und in der frühen Neuzeit" wurde im Herbst 2002 im Institut für Geschichte und Archivkunde der Nikolaus Kopernikus Universität in Toruń unter der Leitung von Professor Roman Czaja begonnen. Das Ziel des Projektes ist es darzustellen, wie das Phänomen Infamie - sowohl im Sinne von ,Strafe', wie auch von ,üblem Ruf' (Leumund) - in den Städten Preußens, Livlands und Nordwestrusslands in der Zeit zwischen dem 13. Jahrhundert und der Mitte des 16. Jahrhunderts funktionierte. Die im Rahmen des zu besprechenden Projektes durchgeführten Forschungen konzentrieren sich auf Gruppen der sozial Geächteten (Randgruppen), die in diesen Städten lebten. Ursachen sozialer Ausgrenzung und der Stigmatisierung mancher sozialen Gruppen bzw. einzelner Individuen werden analysiert, wie auch Folgen dieses Phänomens, die sich in spezifischen Existenzformen dieser Ausgegrenzten, in der Art und Weise der Kontakte zu anderen Mitbewohnern, des Verhältnisses der weltlichen und kirchlichen Behörden zu dem gesamten Personenkreis äußerten.

Die Forschungen konzentrieren sich nicht ohne Grund auf Großstädte wie Kulm/Chełmno, Elbing/Elbląg, Danzig/Gdańsk, Königsberg/Króle-

* Material für den vorliegenden Beitrags habe ich während meines Aufenthaltes in Marburg (in der Zeit vom Oktober bis Dezember 2003, im Rahmen eines vom Herder-Institut gewährten Stipendiums) und in Hamburg (Oktober 2004 - Februar 2005, im Rahmen eines von der ZEIT-Stiftung gewährten Stipendiums) zusammengestellt; wofür ich mich an dieser Stelle bei den beiden Institutionen sehr herzlich für die erwiesene Unterstützung bedanken möchte. 
wiec/Kaliningrad, Thorn/Toruń, Reval/Tallinn, Riga, Nowgorod und Pleskau/Pskow. Zum Ersten waren das in der zu besprechenden Periode große städtische Zentren - manche davon sogar im europäischen Maßstab, und zwar sowohl im Hinblick auf die Anzahl der dort bewohnenden Bevölkerung wie auch auf ihre Handels-, Wirtschafts- und kulturelle Bedeutung. Die Geächteten und Ausgegrenzten, die dort lebten, lebten nach anderen Prinzipien als in kleineren Zentren, sie waren durch größere Mobilität, eine kompliziertere innere Struktur ihrer Gruppierungen, und auch durch ihre Art der Lebensführung charakterisiert. Zweitens werden die Forschungen zu den genannten Städten durch eine relativ gute Überlieferung erleichtert, obwohl sie im Falle von Nowgorod und Pskow einen anderen Charakter hat, was den Einsatz anderer Forschungsmethoden erfordert.

Der geographische Rahmen wurde gewählt, um Merkmale des Phänomens des „üblen Rufes“ in zwei verschiedenen Kulturkreisen - dem lateinischen und byzantinischen - zu vergleichen. Obwohl das Vorhaben viele Schwierigkeiten bereitet - vor allem wegen der beträchtlichen Unterschiede im Charakter und Erhaltungszustand der Überlieferung - ist es durch die allgemeine Verbreitung - auch im byzantinischen Kulturkreis sowohl des Begriffes des ,üblen Rufes“ wie auch der Infamie als Strafe (Ächtung/Acht, Bann) begründet.

Da Veränderungen in der menschlichen Mentalität nur dann festgestellt werden können, wenn entsprechend lange Zeitperioden untersucht werden, so wurde auch in unserem Projekt ein relativ breiter chronologischer Rahmen zugrunde gelegt. Dank dieser Maßnahmen wurde auch die Möglichkeit geschaffen, Beobachtungen über Auswirkungen der Reformation in den preußischen und livländischen Städten sowie der Veränderungen auf der politischen Karte (Anschluss von Nowgorod, später von Pskow an das Großherzogtum Moskau), die sich um die Wende des 15. zum 16. Jahrhundert auf dem Gebiet Nordwestrusslands vollzogen, auf den Inhalt des Begriffes des „üblen Rufes“ anzustellen. Der formal abschließende Zeitpunkt - Mitte des 16. Jahrhunderts - hat jedoch in vielen Fällen nur einen symbolischen Charakter. Weil die erhaltene Überlieferung sehr bruchstückhaft ist, können viele Phänomene erst durch Hinzuziehung späteren neuzeitlichen Materials richtig eingeordnet werden.

Da der vorgegebene Umfang der vorliegenden Skizze mich zwang, eine eng begrenzte Frage zur Präsentation auszuwählen, entschied ich mich, einige Bemerkungen zu einem spezifischen Amt der Stadtverwaltung zu präsentieren, das eine unanfechtbare Bedeutung für das ordnungsgemäße 
Funktionieren der Stadt aber gleichzeitig einen „üblen Ruf " hatte, und im Zusammenhang damit an den Rand des gesellschaftlichen Lebens gedrängt wurde: Das Scharfrichter- oder Henkeramt, das sich einer großen Popularität bei Forschern erfreut, die sich mit Geächteten (im weiten Sinn) aller Epochen befass(t)en und zwar vom Altertum bis in die Neuzeit.' Die von mir eingangs erwähnte Beschränkung des Umfangs der vorliegenden Skizze ist der Grund, weshalb die hier vorgetragenen Bemerkungen auf preußische und livländische Städte begrenzt bleiben.

Der Scharfrichter erscheint in livländischen Städten früher als in preuBischen. In Reval wird er zum ersten Mal in den dreißiger Jahren des 14 . Jahrhunderts erwähnt (tortor) ${ }^{2}$, in Riga wahrscheinlich schon am Anfang

' Z. B.: Werner DANCKERT. Unehrliche Leute. Die verfemten Berufe, Bern 1963, S. 23-49; Franz IRSigler, Arnold Lassota, Bettler und Gaukler. Dirnen und Henker. Randgruppen und Außenseiter in Köln 1300-16(00. Köln 1984, S. 228-282; Heribert AIGENER. Zur gesellschaftlichen Stellung von Henkern, Gladiatoren und Berufsathleten, in: Soziale Randgruppen und Außenseiter im Altertum. Referate vom Symposion ,Soziale Randgruppen und antike Sozialpolitik" in Graz (21. bis 23. September 1987), hg. v. Ingomar WEILER. unter der Mitwirkung v. Herbert Grassl, Graz 1988, S. 201-220; Werner PETERS, Der Fronbote als Nachrichter. Überlegungen zu seiner Darstellung in den Codices picturati des Sachsenspiegels, in: Der Sachsenspiegel als Buch (Germanistische Arbeiten zu Sprache und Kulturgeschichte, Bd. 1), hg. v. Ruth SCHMIDT-WIEGAND. Dagmar HüPPER, Frankfurt am Main 1991, S. 295-314; Jutta NowoSADTKO, Scharfrichter und Abdecker. Der Alltag zweier „unehrlicher Berufe" in der Frühen Neuzeit, Paderborn 1994; Gisela WILBERTZ, Scharfrichter und Abdecker. Aspekte ihrer Sozialgeschichte vom 13. bis zum 16. Jahrhundert. in: Randgruppen der spätmittelalterlichen Gesellschaft. Ein Hand- und Studienbuch, hg. v. Bernd-Ulrich HERGEMÖLLER, Warendorf ${ }^{4}$ 1994, S. 121-156; Wolfgang SCHEFFKNECHT, Scharfrichter. Vom römischen carnifex bis zum frühneuzeitlichen Staatsdiener, in: Randgruppen der spätmittelalterlichen Gesellschaft, hg. v. Bernd-Ulrich HERGEMÖLLER, Warendorf 2001, S. 122-172; in der polnischen Historiographie die Monographie von Hanna ZAREMSKA, Niegodne rzemiosło. Kat w społeczeństwie Polski XIV-XVI wieku [Das unehrliche Handwerk. Scharfrichter in der Gesellschaft Polens, 14.-16. Jh.J, Warszawa 1986 und geringe Beiträge, z. B.: Witold Maisel. Sądownictwo miasta Poznania do końca XVI wieku [Gerichtsbarkeit der Stadt Posen bis zum Ende des 16. Jahrhunderts], Poznań 1961, S. 232-238; Stanisław WAŁȨGA, Kuracje katowskie i opresje akuszerskie $w$ dawnym Toruniu [Henkerstherapien und Zwangslagen der Hebammen in altem Thorn], in: Rocznik Toruński, Bd. 5: 1971, S. 65-85; DERS., O katach, hyclach i oprawcach w dawnym Toruniu [Über Henker, Hundefänger und Henkersknechte im alten Thorn], in: ebd., Bd. 10: 1975, S. 297-303; Radosław GAZIŃsKI, Urząd kata w Gorzowie w II polowie XVII i XVIII w. [Henkersamt in Landsberg in der 2. Hälfte des 17. Jahrhunderts und im 18. Jahrhundert], in: Nadwarciański Rocznik Historyczno-Archiwalny, 2002, Nr. 9, S. 79-87, sowie die Studien von Dariusz KACZOR, Andrzej KARPIŃSKI und Jacek WIJACZKA in: KwartHKM, 2005, Nr. 3-4; siehe auch: Maciej TRZCIŃ́sKI. Miecz katowski. Prȩgierz. Szubienica. Zabytki jurysdykcji karnej na Dolnym Sląsku (XIII-XVIII w.) [Henkersschwert. Pranger. Galgen. Denkmäler der Strafjurisdiktion im Niederschlesien], Wroclaw 2001.

${ }^{2}$ Liv-, Esth- und Curländisches Urkundenbuch nebst Regesten, hg. v. Friedrich Georg VON BunGE, [Abt. 1], Bd. 2: 1301-1367, Reval 1885. Nr. CMXXIV, Sp. 486; Libri de diversis articulis 1333-1374, hg. v. Paul JOHANSEN (Publikationen aus dem Revaler Stadtarchiv, Nr. 8), Reval 1935, S. 127. 
der 2. Hälfte des 14. Jahrhunderts. ${ }^{3}$ Ein Scharfrichter im Dienste der Danziger Behörden wird ebenfalls in der 2. Hälfte des 14. Jahrhunderts urkundlich bestätigt, ${ }^{4}$ und in der Altstadt Thorn wird er zum ersten Mal 1401 erwähnt. ${ }^{5}$ In der Altstadt Elbing wurde der Abdecker (also entweder Helfer des Scharfrichters oder er selbst) bereits 1411 urkundlich erwähnt. ${ }^{6}$ Ein gewisses Problem stellt Kulm dar, denn bereits 1407 wandten sich seine Behörden an den Thorner Rat mit dem Ersuchen, den Scharfrichter zwecks Durchführung einer Hinrichtung zu schicken. ${ }^{7}$ Auf dieser Grundlage kann man schlussfolgern, dass der Rat von Kulm schon damals keinen eigenen Henker unterhielt, was wiederum durch die Krise bedingt war, deren erste Symptome in Kulm bereits in der 2. Hälfte des 14. Jahrhunderts festzustellen sind und die sich zu Anfang des 15. Jahrhunderts verstärkten. ${ }^{8}$

\footnotetext{
${ }^{3}$ Kämmerei-Register der Stadt Riga 1348-136 und 1405-1474, bearb. v. August vON BULMERINCQ, Bd. 1, Leipzig 1909. S. 49.

${ }^{4}$ Joachim ZDRENKA, Regesty dokumentów zachodniopomorskich w archiwum gdańskim [Regesten der westpommerischen Urkunden im Danziger Archiv], in: Informator Archiwalny WAP w Szczecinie, Nr. 8 (Dezember 1981), Nr. 83 (1376-1400).

${ }^{5}$ Thorner Denkwürdigkeiten von 1345-1547, hg. v. Albert VolGT, in: Mitteilungen des Coppernicus-Vereins für Wissenschaft und Kunst zu Thorn. H. 13: 1904, S. 27; im 15. Jh. wird er regelmäßig erwähnt; im Staatsarchiv Thorn befinden sich zahlreiche Schreiben der anderen Städte und Beamten, die um Überlassung des Thorner Scharfrichters bitten; z. B.: Archiwum Państwowe w Toruniu, Katalog I, Nr. 1197 (Bitte des Stadtrates Brombergs, 1. Hälfte des 15. Jh.s); Nr. 1060 (Bitte des Ordenskomturs von Schönsee, sicher Anfang des 15. Jahrhunderts, vor Ende Novembers 1416; Die Komturei Schönsee wurde wahrscheinlich am 24. November 1416 aufgelöst, vgl. S. Jóźwiak. Zmiany w sposobie funkcjonowania administracji terytorialnej $w$ ziemi chełmińskiej pod panowaniem zakonu krzyżackiego $w$ latach 1410-1422 [Veränderungen in der Funktionsweise der Territorialverwaltung im Kulmer Land unter der Herrschaft des Deutschen Ordens in den Jahren 1410-1422], Zapiski Historyczne 67, 2002, H. 3-4, S. 67); Nr. 2391 (Bitte des Burggrafen von Schwetz, 27. September 1482); Nr. 2406 (Bitte des Stadtrates von Sroda Wielkopolska (?), 5. März 1483); Nr. 2347 (Bitte des Starosts von Roggenhausen, 5. Oktober 1483).

${ }^{6}$ Nowa Księga Rachunkowa Starego Miasta Elbląga 1404-1414 [Das neue Rechnungsbuch der Altstadt Elbing, 1404-1414], hg. v. Markian PELECH, T. 1 (1404-1410), Warszawa 1987, Nr. 614, S. 131; T. 2 (1411-1414), Warszawa 1989, Nr. 1125, s. 11; siehe auch: ebd., T. 2, Nr. 1265, S. 38 (1412); Nr. 1341, S. 60 (1413); Nr. 1460, S. 87 (1414) - Ausgaben aus der Stadtkasse für „richteswerte“.

${ }^{7}$ Archiwum Państwowe w Toruniu, Katalog I, Nr. 596 (26. Juni 1407).

${ }^{8}$ Sehe dazu: Zenon NowaK, Dzieje Chełmna do końca XVIII wieku [Die Geschichte Kulms bis zum Ende des 18. Jahrhundertsl, in: Dzieje Chełmna i jego regionu. Zarys monograficzny, hg. v. Marian BiskuP, Toruń 1986, S. 121, 137-138; Roman CzAJA, Spór cechów chełmińskich z radą miejską. Przyczynek do konfliktów społecznych w miastach pruskich w średniowieczu [Der Streit der Kulmer Zünfte mit dem Stadtrat. Ein Beitrag zu den Sozialkonflikten in preußischen Städten in Mittelalter], in: Prusy-Polska-Europa. Studia 2 dziejów średniowiecza i czasów wczesnonowożytnych. Prace ofiarowane Profesorowi Zenonowi Hubertowi Nowakowi w sześćdziesiątạ piạtą roczniç urodzin i czterdziestolecie pracy naukowej, hg. v. Andrzej RADZIMIŃSKI u. Janusz TANDECKI. Toruń 1999, S. 332.
} 
Die frühere Erwähnung des Scharfrichters in den Revaler und Rigaer Quellen kann man vor allem mit dem breiteren Umfang der Gerichtsbarkeit erklären, die diese Städte innehatten. Die Behörden von Reval, wie auch Riga, hatten bereits im 13. Jahrhundert das Recht zur Vollstreckung der Todesstrafe, auch in Bezug auf Straftaten, die in den Grenzen des Landgebietes der Städte verübt wurden. Zwar versuchte der Deutsche Orden im 14 Jahrhundert Riga das Recht auf hohe Gerichtsbarkeit einzuschränken, doch schließlich verzichtete er auf seine Forderungen. ${ }^{9}$ Dagegen begannen die Stadtgemeinden in Preußen erst viel später mit ihren Bemühungen, das Recht auf Ausübung der Hochgerichtsbarkeit zu erlangen. Zwar konnten sie in der zweiten Hälfte des 13. Jahrhunderts die ersten Erfolge verbuchen, doch wurde z. B. die hohe Gerichtsbarkeit im Landgebiet in den meisten Fällen erst im 14. Jahrhundert von ihnen übernommen. ${ }^{10}$ Unter Berücksichtigung der oben dargelegten Spezifik kann hypothetisch vorausgesetzt werden, dass sowohl die Behörden von Reval, wie auch von Riga bereits im 13. Jahrhundert eigene Scharfrichter unterhalten konnten. Dagegen entstand in den preußischen Städten der Bedarf, deren Dienste ständig in Anspruch zu nehmen, frühestens am Ende des 13. Jahrhunderts.

Der üble Ruf des Henkers beruhte vor allem auf der Art seiner Pflichten. Im lateinischen Europa gehörte im allgemeinen dazu u. a. die Vollstreckung der ausgesprochenen Todesstrafen (einschließlich verschiedener Formen der Hinrichtungen), die Durchführung von Folterungen (ihre Bedeutung wuchs allmählich, angefangen mit dem 16. Jahrhundert), sowie die Erfüllung verschiedenartiger Ordnungsfunktionen (Sanitärfunktionen). ${ }^{11}$

\footnotetext{
${ }^{9}$ Friedrich Georg vON BUNGE, Die Stadt Riga im dreizehnten und vierzehnten Jahrhundert. Geschichte, Verfassung und Rechtszustand, Leipzig 1878, S. 18-49, 81-83; Constantin METTIG, Geschichte der Stadt Riga, Riga 1897, S. 39-68; August von BulmerincQ, Die Verfassung der Stadt Riga im ersten Jahrhundert der Stadt. Ein Beitrag zur Geschichte der deutschen Stadtverfassung, Leipzig 1898, S. 85-97; Manfred HELLMANN, Der Deutsche Orden und die Stadt Riga, in: Stadt und Orden. Das Verhältnis des Deutschen Ordens zu den Städten in Livland, Preußen und im Deutschen Reich, hg. v. Udo ARNOLD (Quellen und Studien zur Geschichte des Deutschen Ordens, Bd. 44), Marburg 1993, S. 1-33; Reinhard VOGELSANG, Reval und der Deutsche Orden: Zwischen städtischer Autonomie und landesherrlicher Gewalt, in: ebd., S. 34-58; Juhan KREEM, The Town and its Lord Reval and the Teutonic Order (in the fifteenth Century) (Tallinna Linnaarhiivi Toimetised, Nr. 6), Tallinn 2002, S. 38-39.

${ }^{10}$ Mehr zu diesem Thema: Roman CZAJA, Miasta pruskie a zakon krzyzacki. Studia nad stosunkami między miastem a władzą terytorialną w późnym średniowieczu [Die preußischen Städte und der Deutsche Orden. Eine Studie zu den Beziehungen zwischen Stadt und Landesherrschaft im späten Mittelalter], Toruń 1999, S. 18-27.

${ }^{11}$ SCHEFFKNECHT, Scharfrichter (wie Anm. 1), S. $125 \mathrm{ff}$.; ZAREMSKA, Niegodne rzemiosło (wie Anm. 1), S. 15-82.
} 
Der volle Umfang und die Art der Tätigkeiten, die die Scharfrichter in den preußischen und livländischen Städten hatten ist erst aus dem 17. und 18. Jahrhundert bekannt. Aus dieser Zeit stammen zahlreiche Verträge, die zwischen dem Scharfrichter und den Stadtbehörden geschlossen wurden, die Auskunft über die Höhe seines festen Gehaltes geben, wie auch über die Beträge, die dem Henker für einzelne Hinrichtungsarten gezahlt wurden, über Gebühren, die er für die Säuberung der Stadtbereiche mit öffentlichem Charakter bezog, für Kloakenreinigung - auch in Privathäusern Entsorgung von Verunreinigungen aus der Stadt, Fangen und Töten von herrenlosen Hunden, Beerdigung von Leichnamen der als ,unehrlich“ erkannten Personen (Selbstmörder, Verbrecher usw.). Die Verträge enthalten auch Informationen über die ihm zustehenden Vorteile (das Recht auf „Dienstwohnung“, Zuteilung von Bekleidung, Lebensmittel). ${ }^{12}$

Ein Teil der vom Scharfrichter im 17. und 18. Jahrhundert wahrgenommenen Aufgaben gehörte schon früher zu seinen Pflichten. Im 14. Jahrhundert in livländischen Städten, ${ }^{13}$ und seit dem Ende dieses Jahrhunderts in preußischen Städten, ${ }^{14}$ macht sich das steigende Interesse der Stadtbehörden für den Sanitärzustand der von ihnen verwalteten Zentren bemerkbar. Obwohl die städtischen Behörden bemüht waren, die Bewohner der Städte mit einem Teil der damit verbundenen Pflichten zu belasten, ${ }^{15}$

${ }^{12}$ Siehe u. a. Latvijas Valsts vēstures arhīvs [Das lettische staatliche historische Archiv], Rigas senatnes un vēstures pêtītāju biedrība, Fonda Nr. 4038, Apr. Nr. 2, Nr. 180 (Urkunden aus den Jahren 1778-1792, die mit den Berufstätigkeiten des Scharfrichters Friedrich Wilhelm Stoff verknüpft sind); Aktenstücke und Urkunden zur Geschichte der Stadt Riga 1710-1740, hg. aus dem Nachlass des Anton BuchHOLTZ durch August VON BULMERINCQ, Bd. I: 1710-1725, Riga 1902, Nr. 325, S. 224-225; Nr. 630, S. 429; Nr. 697, S. 507: Nr. 420. S. 356; Bd. 2: 1725-1740. Riga 1903, Nr. 491, S. 425 (1734); Otto GüNTHER. Ein Kontrakt mit dem Scharfrichter von Dirschau, in: Mittheilungen des Westpreußischen Geschichtsvereins I, 1902, Nr. 3, S. 58-60; Paul SiMSON, Geschichte der Danziger Willkür. Danzig 1904. S. 151. 163; Stanisław WAŁ.ȨGA. Kuracje katowskie (wie Anm. 1), S. 67-68; DERS., O katach (wie Anm. 1), S. 297-303; Martina THOMSEN, Zwischen Hauptwache und Stockhaus. Kriminalität und Strafjustiz in Thorn im 18. Jahrhundert (Materialien und Studien zur Ostmitteleuropa-Forschung, 13), Marburg 2005, S. 68-77.

${ }^{13}$ Siehe z. B.: Liv-, Esth- und Curländisches Urkundenbuch, [Abt. 1], Bd. 2 (wie Anm. 2), Nr. CMXXIV, Sp. 489 (1345); vgl. Paul JohANSEN, Heinz von ZUR MÜHLEN, Deutsch und Undeutsch im mittelalterlichen und frühneuzeitlichen Reval (Ostmitteleuropa in Vergangenheit und Gegenwart, 15), Köln 1973, S. 241-243.

${ }^{14}$ Roman CZAJA, Troska o stan sanitarny w miastach pruskich w XIV i XV w. [Sorge um den Sanitärzustand in preussischen Städten im 14. und 15. Jahrhundert]. in: KwartHKM. 2005, Nr. 3-4, S. 343-349.

${ }^{15}$ Als Beispiele siehe: Georg BENDER, Die ältesten Willküren der Neustadt Thorn. in: Zeitschrift des Westpreußischen Geschichtsvereins 7, 1882, S. 112 (Artikel 27): Arthur SEMRAU, Die Willkür der Stadt Kulm von etwa 1400, in: Mitteilungen des Coppernicus-Vereins für Wissenschaft und Kunst zu Thorn 35, 1927, S. 45 (Artikel 30); JOHANSEN, vON ZUR MÜHLEN, Deutsch und Undeutsch (wie Anm. 13). S. 437 (Revaler Burskprake aus dem Anfang des 15. Jahrhunderts). 
waren sie angesichts der innerhalb der Stadtmauer vorhandenen Bereiche öffentlichen Charakters und der allgemeinen Abneigung der Einwohner, manche Ordnungstätigkeiten selbst auszuführen (z. B. Kloakenreinigung), gezwungen, Personen zu finden, die zur Ausführung dieser erniedrigenden Aufgaben bereit waren. Ähnlich, wie in den westeuropäischen Städten, ${ }^{16}$ nahm auch in Preußen und Livland der Scharfrichter diese Pflichten wahr, meistens in der Begleitung von Vertretern anderer Gruppen mit niedrigem sozialen Status - Tagelöhner, Stadtknechte, und seit dem 16 Jahrhundert auch Verbrecher, die ihre Strafeverbüßten. ${ }^{17}$ Bei der Reinigung der Aborte (diese Beschäftigung wurde als besonders ehrenrührig angesehen), wurde der Scharfrichter meistens durch einen seiner Gehilfen (die als Abdecker bezeichnet wurden) vertreten ${ }^{18}$. Der Henker (oder Abdecker) in der Rolle des Straßenfegers oder Kloakenentsorgers erscheint in Reval im 15. Jahrhundert, ${ }^{19}$ doch wahrscheinlich war er in dieser Eigenschaft bereits im vorhergehenden Jahrhundert tätig. In Elbing stammen die ältesten Erwähnungen darüber aus dem Anfang des 15. ${ }^{20}$ in Danzig aus der ersten Hälfte des 16. Jahrhunderts, ${ }^{21}$ dagegen in Thorn aus der zweiten Hälfte des 16. Jahrhunderts. ${ }^{22}$

$\mathrm{Zu}$ den Pflichten des Abdeckers gehörte auch das Fangen und Töten von Hunden. Die ältesten Erwähnungen über solche Aufgaben der Abdecker in preußischen und livländischen Städten stammen erst aus der

${ }^{16}$ Irsigler, Lassota, Bettler und Gaukler (wie Anm. 1), S. 270-282; NowOSADTKo, Scharfrichter und Abdecker (wie Anm. 1), S. 142-152.

${ }^{17}$ Archiwum Państwowe w Toruniu, Katalog II, Abteilung VIII, 14, S. 6 (1595); Archiwum Państwowe w Gdańsku [Staatsarchiv Danzig]. 300, 12/2, S. $433-435$ (1532); D. Janicka, Prawo karne w trzech rewizjach prawa chełmińskiego z XVI wieku [Strafrecht in drei Revisionen des Kulmer Rechtes aus dem 16. Jahrhundert]. Toruń 1992, S. 54.

${ }^{18}$ Natürlich erfolgte diese Teilung der Pflichten nur in diesen Zentren, die imstande waren. neben dem Scharfrichter auch Gehilfen für ihn anzustellen, wie z. B. in der 2. Hälfte des 14. Jh.s in Reval (Küllike KAPLINSKI, Tallinna käsitöölised XIV sajandil [Revals Handwerker im 14. Jahrhundert], T. 2, Tallinn 1980, S. 31 - 1374, Laurentius ,rakker“ wohnt im Jahre 1374 zusammen mit Hinzo Brunswik; Hinzo Brunswik ist zweifellos identisch mit Hincken „scharprichtere“, der in den Stadtrechnungen aus dem Jahre 1376 und 1378 erwähnt wird: Das Revaler Kämmereibuch von 1376 bis 1380, bearb. v. Dieter HECKMANN. ZfO, Jg. 4l, 1992, H. 2, S. 206, 224.

${ }^{19}$ Siehe z. B.: Kämmercibuch der Stadt Reval 1432-1463, bearb. v. Reinhard VogelsanG, Erster Halbband Nr. 1-769 (QDhG, N.F., Bd. XXIV/1), Köln 1976, Nr. 524, S. 253; Kämmereibuch der Stadt Reval 1463-1507, bearb. v. Reinhard VoGELSANG, Zweiter Halbband Nr. 1991-2754 (QDhG, N. F., Bd. XXVIl/2), Köln 1983, Nr. 2080, S. 533; Nr. 2153, S. 576.

${ }^{20}$ Nowa Księga Rachunkowa Starego Miasta Elbląga (wie Anm. 6), T. 2, Nr. 1175, S. 11.

${ }^{21}$ Archiwum Państwowe w Gdańsku. 300, 12/2, S. 76. 295.

${ }^{22}$ Archiwum Państwowe w Toruniu, Katalog II. Abteilung X, 2, S. 76, 78; Miscellanea źródłowe do historii kultury i sztuki Torunia [Quellenmiscellanea zur Geschichte der Kultur und Kunst Thorns], bearb. v. Bogusław DYBAŚ, Marek FARBISZEwSKI (Źródła i materiały do dziejów sztuki polskiej, Bd. 22), Wrocław 1989, S. 15-16 (1565-1566). 
ersten Hälfte des 16. Jahrhunderts, ${ }^{23}$ obwohl gewisse topographische Bezeichnung nahe legen, dass schon früher regelmäßig Kadaver aus diesen Städten entfernt wurden. ${ }^{24}$

$\mathrm{Zu}$ dem schlechten Ruf des Henkers hatte auch die Pflicht zur Beerdigung von Leichnamen der Menschen beigetragen, die nicht in geweihtem Boden beigesetzt werden durften (hauptsächlich Selbstmörder und hingerichtete Verbrecher). ${ }^{25}$ Den frühesten Beleg für diese Pflicht findet man in den Revaler Quellen (2. Hälfte des 15. Jahrhunderts), ${ }^{26}$ dagegen stammen die ersten Spuren für diese Betätigung in den preußischen Städten erst aus dem 16. Jahrhundert. ${ }^{27}$ Auch in diesem Fall nahm der Scharfrichter die Aufgaben meistens nicht persönlich wahr, sondern ließ sich durch seine Gehilfen vertreten.

Das Verhältnis des Durchschnittbürgers und der Behörden der preußischen und livländischen Städte zur Person des Scharfrichters und zu den sich in diesem Amt vollziehenden Veränderungen ist wegen des Fehlens einer entsprechenden Quellenbasis sehr schwer nachzuvollziehen. Die im 17. und 18. Jahrhundert deutlich fassbare Abneigung oder sogar Feindseligkeit, ${ }^{28}$ hat zweifelsohne eine viel längere Geschichte, geht jedoch

${ }^{23}$ Z. B. wurde in Danzig am 13. Mai 1531 dem Abdecker der Lohn für die Tötung von 420 Hunden gezahlt (Archiwum Państwowe w Gdańsku, 300, 12/2, s. 298); siehe auch: Max FolTZ, Geschichte des Danziger Stadthaushalts, Danzig 1912, S. 153; vgl. Otto BRUnNER, Die Finanzen der Stadt Wien von den Anfängen bis ins 16. Jahrhundert (Studien aus dem Archiv der Stadt Wien, Bd. 1/2). Wien 1929, S. 221.

${ }^{24}$ Man vergleiche z. B. die Bezeichnung ,.Schindegraben', die in der Vorstadt der Neustadt Thorn schon am Ende des 14 Jh.s erwähnt wurde; Księga ławnicza Nowego Miasta Torunia (1387-1450) [Schöffenbuch der Neustadt Thorn (1387-1450)], hg. v. Karola CiESIELSKA (Towarzystwo Naukowe w Toruniu, Fontes 63), Warszawa 1973, Nr. 134. S. 18; Nr. 1016, S. 100; Nr. 1127, S. 111; Nr. 1151, S. 115; Nr. 1205, S. 122; Nr. 1345, S. 147; Nr. 1418 , S. 160; Nr. 1543, S. 185; Nr. 1697. S. 206; Nr. 1699. S. 206; Nr. 1800, S. $218 ;$ Nr. 2046. S. 251; Jerzy PRZERACK1, Księga ławnicza sạdu przedmiejskiego Nowego Miasta Torunia z lat 1444-1457 [Schöffenbuch des vorstädtischen Gerichtes der Neustadt Thorn aus den Jahren 1444-1457], in: Studia ofiarowane Profesorowi Antoniemu Czacharowskiemu w sześćdziesiąaţ pią̧ą̧ rocznicę urodzin i czterdziestolecie pracy naukowej (Studia Polonica Historiae Urbanae, Bd. 2), Toruń 1996, Nr. 6, S. 317: Nr. 11, S. 318; Nr. 26, S. 320; Nr. 37, S. 322: Nr. 48-49, S. 324: Nr. 64, 67. S. 326; Tabliczki woskowe miasta Torunia ok. 1350 - I poł. XVI w. [Wachstafeln der Stadt Thorn, ca. 1350 - 1. Hälfte des 16. Jahrhunderts], hg. v. Karol GóRSKI. Witold SzCZUCZKO, Warszawa 1980, S. 118, 120, 154.

${ }^{25}$ Jürgen DieselhoRST, Die Bestrafung der Selbstmörder im Territorium der Reichsstadt Nürnberg, in: Mitteilungen des Vereins für Geschichte der Stadt Nürnberg, Bd. 44, 1953 (Festschrift des Vereins für Geschichte der Stadt Nürnberg zur Feier seines fünfundsiebzigjährigen Bestehens, 1878-1953), S. 64-67. 71-73. 96-97.

${ }^{26}$ Eugen vON NOTTBECK, Die alte Criminalchronik Revals, Reval 1884, S. 29, 54; Die Quellen des Rigischen Stadtrechts bis zum Jahr 1673, hg. v. Jakob Gottlieb Leonhard NAPIERSKY, Riga 1876, S. 193 (Statut Nr. X: „Van roveren vnde van deven“, Artikel 8).

${ }^{27}$ Max FolTz, Der Danziger Stadthaushalt am Ende des 16. Jahrhunderts, in: Zeitschrift des Westpreußischen Geschichtsvereins, H. 49, 1907, S. 155.

${ }^{28}$ THOMSEN. Hauptwache (wie Anm. 12), S. 69 ff. 
nicht über das 14. Jahrhundert zurück. Dies beweist die Überlieferung in Reval. Zwar lassen sich bereits in der ersten Hälfte des 14 Jahrhunderts auch hier gewisse Anzeichen der Abneigung gegenüber der Person des Henkers verzeichnen (ihre tatsächlichen Ursachen sind jedoch unklar), ${ }^{29}$ doch wird der Scharfrichter noch $1374^{30}$ als Mitbewohner (gemeinsam mit dem Abdecker) der Immobilie auf dem Gelände der St. Olafpfarrei erwähnt; auch entrichtete er wie die anderen Schoß (dies ist eine direkte Bestätigung der Zugehörigkeit zur Gemeinschaft). Besonders erwähnenswert ist, dass der damalige Scharfrichter (Hinzo Brunswik) den Namen einer der Revaler Familien trug, deren Vertreter in der ersten Hälfte des 14. Jahrhunderts sogar Ratsherren waren (es wird sich dabei eine zufällige Namensgleichheit handeln). ${ }^{31}$ Später (höchstwahrscheinlich im 15. Jahrhundert) wurde der Sitz des Scharfrichters jedoch in das Gebiet der Nikolauspfarrei verlegt, in die Nähe der städtischen Abdeckerei (oder direkt in diese)..$^{32}$ Es ist jedoch schwer festzustellen, was die Ursachen der Ortsveränderung in Reval waren. Wenn der Scharfrichter, der die Ortsveränderung vornehmen musste, ein Este war, ${ }^{33}$ könnte die seit der Wende des 14. zum 15. Jahrhundert von den Stadtbehörden verfolgte Politik der Einschränkung der Rechte von Esten beim Kauf von Immobilien im Stadtgebiet, ${ }^{34}$ die Annahme einer Wohnung, die ihm von den Revaler Behörden angeboten wurde, erzwungen worden sein. Es ist bemerkenswert, dass seit dem 15. Jahrhundert die Scharfrichter in den meisten preußischen und livländischen Städten Wohnungen hatten, die Eigentum der Gemeinde waren. Ihre Lage - meistens in einem der Türme im städtischen Befestigungsring, bzw. ein direkt an die Stadtmauer anschließendes Gebäude spiegelt die spezifische Position des Scharfrichters. ${ }^{35}$ Er blieb weiterhin

${ }^{29} \mathrm{Vgl}$. Johansen, VON ZUR MüHLEn, Deutsch und Undeutsch (wie Anm. 13), S. 239.

${ }^{30} \mathrm{Im}$ Schossbuch wurde nicht angegeben, ob er damals die Rolle des Scharfrichters ausübte; siehe Anm. 18.

${ }^{31}$ Friedrich Georg VON BUNGE, Die Revaler Rathslinie nebst Geschichte der Rathsverfassung und einem Anhange über Riga und Dorpat, Reval 1874, S. 84.

32 Paul Johansen, Heinz von ZUR MÜHLEN, Deutsch und Undeutsch (wie Anm. 13). S. 44.

${ }^{33}$ Neben Scharfrichtern - Deutschen, gab es in Reval auch Henker-Esten; Heinz vON ZUR MÜHLEN, Siedlungskontinuität und Rechtslage der Esten in Reval von der vordeutschen Zeit bis zum Spätmittelalter, in: ZfO 18, 1969, H. 4, S. 647; JOHANSEN, VON ZUR MÜHLEN, Deutsch und Undeutsch (wie Anm. 13), S. 240.

${ }^{34}$ Vilho NitTemaA, Die undeutsche Frage in der Politik der livländischen Städte im Mittelalter, Helsinki 1949, S. 66-67; Heinz VON ZUR MÜHLEN, Siedlungskontinuität (wie Anm. 33). S. $648 \mathrm{ff}$.

${ }^{35}$ Kämmerei-Register der Stadt Riga (wie Anm. 3), Bd. 1, S. 117 (1416/1417, ,scharprichters torn"); Friedrich BENNINGHOVEN. Das Stadtbuch von Schwetz 1374-1454, in: ZFO 21 , 1972. H. 1, S. 62 (1441); Krzysztof MiKULSKI, Przestrzeń i społeczeństwo Torunia od końca XIV do początku XVIII wieku [Der Raum und die Gesellschaft Thorns von der Mitte des 14. 
ein Stadtdiener und erhielt aus diesem Grund die Wohnung innerhalb der Stadtmauern, doch gleichzeitig gehörte er zu der Gruppe der Ausgegrenzten, und dies wiederum war der Anlass, seine Person an den Rand des Gemeindelebens zu verdrängen (also in die Nähe der Befestigungen). Aufgrund der hier gemachten Bemerkungen kann die Schlussfolgerung gezogen werden, dass die bereits im 14. Jahrhundert zu beobachtenden Anzeichen der Abneigung gegenüber der Person des Scharfrichters sich in Preußen und Livland im nächsten Jahrhundert zu konkreten Maßnahmen der Ausgrenzung auswuchsen.

\section{III}

Zum Schluss muss dem „schlechten Ruf“ der Orte ein wenig Aufmerksamkeit gewidmet werden, die mit der Arbeit des Scharfrichters verbunden waren, und zwar dem der Galgenberge. Der negative Ruf dieser Objekte war die Folge von deren Bestimmung: hier wurden entehrende Strafen vollzogen (u. a. durch Hängen), hier wurden die Leichen der Gehenkten zur Schau gestellt, am Fuße des Galgens wurden Selbstmörder und hingerichtete Verbrecher beerdigt. In der unmittelbarer Nähe des städtischen Galgens wurden auch Schindergruben („Schinderkaulen“; „Schinderkuhlen“) angelegt, in welche die aus der Stadt entsorgten Tierkadaver und auch Leichen von Menschen, die der traditionellen Beerdigung unwürdig befunden wurden, geworfen wurden (solche Gruben gab es in der Nähe des Galgens u. a. in Königsberg, ${ }^{36} \mathrm{Riga}^{37}$ und Reval ${ }^{38}$ ). Aus diesen Gründen befanden sich die städtischen Galgen bei allen preußischen und livländischen Städten außerhalb der Stadtmauer, ${ }^{39}$ mit dem deutlichen Hang zu den Grenzen des städtischen Landgebietes hin.

Jahrhunderts bis Anfang des 18. Jahrhunderts], Toruń 1999, S. 381; vgl. H. ZAREMSKA, Niegodne rzemiosło (wie Anm. 1), S. 28.

${ }^{36}$ Fritz Gause, Die Geschichte der Stadt Königsberg in Preussen, Bd. 2: Von der Königskrönung bis zum Ausbruch des Ersten Weltkrieges, Köln ${ }^{2} 1996$, S. 26.

${ }^{37}$ Latvijas Valsts vēstures arhīvs, Rigas Senatnes un Vēstures Pētītäju Biedrība, Fonda Nr. 4038, Apr. 2, Nr. 383: „Manuscripta, varia zur Geschichte des 16. u. 17. Jahrhunderts (Geschenk des Oberpastors M. Thiel v. November 1839)", fol. 47; Das Neue Landbuch 1494-1693, in: Vier Bücher der Landvogtei der Stadt Riga, bearb. v. August voN BuLMERINCQ, Bd. 2, Drittes Buch, Riga 1925 Nr. 35l, S. 72.

${ }^{38}$ VON NOTTBECK. Die alte Criminalchronik Revals (wie Anm. 26), S. 54; JOHANSEN, vON ZUR MÜHLEN, Deutsch und Undeutsch (wie Anm. 13), S. 239.

${ }^{39}$ Vereinzelt errichtete man den Galgen auf städtischen Märkten, doch nur vorübergehend und ihr Bau war mit Hinrichtungen ungewöhnlicher Art verbunden, z. B.: Latvijas Valsts vēstures arhīvs. Rīgas Magistrāta Iekšējais Arhivs, Fonda Nr. 8, Apr. 1, Nr. 24: „Kämmerei Register der Stadt Riga" aus den Jahren 1555-1556, fol. 29. 
Galgenberge (ähnlich auch die Pranger) waren ein Symbol der ausgeübten Gerichtsbarkeit. ${ }^{40}$ Ohne Zweifel erschienen sie in den Städten der preussisch-livländischen Region zum Zeitpunkt der Übernahme der hohen Gerichtsbarkeit im Landgebiet durch die Gemeinde, obwohl sie in den Qucllen relativ spät erwähnt werden (Reval - Galgen bei „Tönnisberg“ erst im 15. Jahrhundert, ${ }^{41}$ andere Hinrichtungsstätten noch später; Riga 16. Jahrhundert, ${ }^{42}$ Altstadt Thorn - zweite Hälfte des 15. Jahrhunderts, ${ }^{43}$ Kulm - 15. Jahrhundert usw. ${ }^{44}$ ) Der symbolische Charakter dieser Orte ist besonders deutlich im Falle des Elbinger Galgens. Der sog. „Galgen Warmiten“, gelegen an der Grenze der Elbing im Privileg von 1246 zuerkannten Gebiete, ${ }^{45}$ erinnerte so an die Rechte des Ordens in Bezug auf die hohe Gerichtsbarkeit und aus diesem Grunde bauten die Behörden der Altstadt Elbing in der ersten Hälfte des 14. Jahrhunderts einen neuen Galgen, der nordwestlich von den altstädtischen Befestigungen stand. ${ }^{46}$

Das auf den Galgenbergen vergossene Blut machte aus diesen ein integrales Element des städtischen Sakralraumes von eindeutig negativer Färbung. ${ }^{47}$ Auch sicherten die spezifischen Merkmale den Galgenbergen gleichermaßen einen festen Platz in den magischen Vorstellungen der Zeitgenossen. So ist auch für die Einwohner der preußischen und livlän-

\footnotetext{
${ }^{40}$ Gerd SCHWERHOFF, Verordnete Schande? Spätmittelalterliche und frühneuzeitliche Ehrenstrafen zwischen Rechtsakt und sozialer Sanktion, in: Mit den Waffen der Justiz. Zur Kriminalitätsgeschichte des Spätmittelalters und der Frühen Neuzeit, hg. v. Andreas BLAUERT, Gerd SCHWERHOFF, Frankfurt am Main 1993, S. 158.

4' Kämmereibuch der Stadt Reval 1463-1507 (wie Anm. 19), Erster Halbband Nr. 1191-1990, Nr. 1423, S. 182.

${ }^{42}$ Latvijas Valsts vêstures arhīvs, Rīgas Magisträta lekšējais Arhivs, Fonda Nr. 8, Apr. 1, Nr. 19: „Liber praefecturae ruralis“, S. 67. 94: Das Neue Landbuch 1494-1693 (wie Anm. 37), Nr. 252, S. 54; Nr. 259-261, S. 56.
}

${ }^{43}$ Księga długów miasta Torunia z okresu wojny trzynastoletniej [Schuldbuch der Stadt Thorn aus der Zeit des dreizehnjährigen Krieges], hg. v. Karola CIESIELSKA, Irena JANOSZBiskupowa (Towarzystwo Naukowe w Toruniu. Fontes 55; Źródla do dziejów wojny trzynastoletniej, Bd. 2), Toruń 1964, Nr. 121, S. 170 (1467).

${ }^{44}$ Geheimes Staatsarchiv Preußischer Kulturbesitz Berlin-Dahlem, XIV. Hauptabteilung, Rep. 322 A, Nr. 4, S. 6, 22, 27, 63, 74, 77; Księga czynszów fary chełmińskiej (1435-1496) [Das Zinsbuch der Kulmer Pfarre (1435-1496)], hg. v. Zenon Hubert NowaK, Janusz TANDECKı (Towarzystwo Naukowe w Toruniu, Fontes 78), Toruń 1994, Nr. 92, S. 10; Księga ławnicza sạdu przedmiejskiego Chełmna 1480-1559 (1567) [Schöffenbuch des Vorstadtgerichts Kulm 1480-1559 (1567)], hg. v. Zenon Hubert NOWAK, Janusz TANDECKI (Towarzystwo Naukowe w Toruniu, Fontes 74), Warszawa 1990, Nr. 114, S. 16.

${ }^{45}$ Preußisches Urkundenbuch. Politische Abtheilung, Bd. 1: Die Bildung des Ordensstaates, 1. Hälfte, hg. v. Rudolf PHILIPPI im Verbindung mit Carl P. WÖLKY, Königsberg in Pr. 1882, Nr. 181, S. 131.

${ }^{46}$ Das Elbinger Stadtbuch. Bd. 1: 1330-1360 (1393), hg. v. Hans W. HoPPE (Zeitschrift für die Geschichte und Altertumskunde Ermlands. Beiheft 3, 1976), Bd. 1, Münster 1976, Nr. 1208, S. 144.

${ }^{47}$ Albrecht KelLER, Der Scharfrichter in der deutschen Kulturgeschichte, Hildesheim 1968, S. 228-237; ZAREMSKA, Niegodne rzemiosło (wie Anm. 1), S. 98. 
dischen Städte der Glaube an die übernatürlichen Eigenschaften dieser Objekte überliefert: Sie wurden zu Orten der Ausübung magischer Praktiken, ${ }^{48}$ in ihrer Nähe siedelten sich Kurpfuscherinnen an, die Menschen zu heilen vorgaben (auch der Henker selbst, der ohne größere Beschränkungen über so grundlegende Bestandteile magischer Mixturen verfügte wie den Henkersstrick, die unter dem Galgen wachsenden Kräuter oder Fragmente von Leichen Verurteilter, beschäftigte sich nicht selten selbst mit medizinischer Behandlung), ${ }^{49}$ es fehlt auch nicht an Fällen der Entwendung von Kleidern der Hingerichteten, die durch den Glauben an ihre magischen Eigenschaften verursacht wurden ${ }^{50}$ usw.

Weil die Galgenberge Beerdigungsstätten von Menschen waren, die aus verschiedenen Gründen gerichtlich als ehrlos erkannt worden waren, wurden dort auch Friedhöfe von ethnischen Gruppen mit niedriger gesellschaftlicher Geltung angelegt. So war es z. B. in Elbing (Pruzzischer Friedhof - „Cymiterium prutenorum“ - auf dem Gelände des „Galgens der Warmiten", später ein Friedhof, der von der Armenbruderschaft genutzt wurde), ${ }^{51}$ und auch in Reval (estisch-schwedischer Friedhof auf dem „Tönnisberg“652). Mit dem schlechten Ruf der Gegenden, in denen sich städtische Galgen befanden, ist auch die Anlage von Leprosorien in ihrer Nähe (u. a. Altstadt Thorn, ${ }^{53}$ Culm, ${ }^{54}$ Alt- und Neustadt Elbing ${ }^{55}$ ) zu verbinden. Die Gesellschaft reagierte auf die Objekte ähnlich wie auf ehrlose

${ }^{48}$ Simon Grunau's Preussische Chronik, hg. v. Max PERLBACH (Die preussischen Geschichtschreiber des XVI. und XVII. Jahrhunderts, Bd. 2), Bd. 2, Leipzig 1889. S. 372-373.

${ }^{49}$ WAŁȨGA, Kuracje katowskie (wie Anm. I), S. 69-79; Ernst GiERlich, Reval 1621 bis 1645: Von der Eroberung Livlands durch Gustav Adolf bis zum Frieden von Brömsebro, Bonn 1991, S. 254, 311.

${ }^{511}$ Eugen VON NOTTBECK, Die alte Criminalchronik Revals (wie Anm. 26), S. 74-75; JOHANSEN, VON ZUR MÜHLEN, Deutsch und Undeutsch (wie Anm. 13), S. 238.

${ }^{51}$ Das älteste Zinsbuch der Altstadt Elbing 1295 bis etwa 1316, hg. v. Arthur SEMRAU, in: Elbinger Jahrbuch 4, 1924. S. 21-22: Michael G. FuCHS. Beschreibung der Stadt Elbing und ihres Gebietes in topographischer, geschichtlicher und statistischer Hinsicht. Bd. 3, Abt. I, Elbing 1826, S. 59-60: Max TOEPPEN, Elbinger Antiquitäten. Ein Beitrag zur Geschichte des städtischen Lebens im Mittelalter, H. 2, Marienwerder 1871, S. 126-127; Roman CZAJA, Socjotopografia miasta Elbląga w średniowieczu [Sozialtopographie der Stadt Elbing im Mittelalter], Toruń 1992, S. 157.

${ }^{52}$ Eugen vON NOTTBECK, Wilhelm NEUMANN, Geschichte und Kunstdenkmäler der Stadt Reval, Zweite Lieferung: Kirchliche Kunst. Die Grabsteine Revals, Reval 1899. S. 122.

${ }^{53}$ Tomasz JASIŃSK1, Przedmieścia średniowiecznego Torunia i Chełmna [Die Vorstädte Thorns und Kulms], Poznań 1982, S. 104-105.

${ }^{54}$ Franz SCHULTZ, Die Stadt Kulm im Mittelalter, in: Zeitschrift des westpreussischen Geschichtsvereins 23, 1888, S. 248; Mariusz HORANIN, Sieć leprozoriów w państwie zakonnym w Prusach [Räumliches Netz von Leprosorien im Ordensstaat in Preussen], in: KMW, 2005. Nr. 2, S. 138.

${ }^{55}$ A. Semrau, Der alte und der neue St. Georg in Elbing, in: Mitteilungen des Coppernicus-Vereins für Wissenschaft und Kunst zu Thorn 30, 1922, S. 60-62. 
Menschen - sie war bemüht, sie an einer Stelle zu konzentrieren und an den Rand der Gesellschaft zu verdrängen (auch im räumlichen Sinne).

Schließlich ist zu bemerken, dass die Galgenberge, die vor den preuBischen und livländischen Städten lagen, auch durch das Prisma der Hinrichtungsstätte gesehen wurden, auf der Jesus Christus gekreuzigt wurde. Das war der Grund für den Brauch, in der Nähe Kapellen anzulegen, die das spezifische Patrozinium ,Jerusalem“ hatten (u. a. bei Reval ${ }^{56}$ und Danzig $^{57}$ ), wo die Verurteilten auf dem Weg zur Hinrichtungstätte sich zum letzten Mal ausruhen bzw. die Beichte ablegen konnten. Mit diesem Assoziationskreis ist auch der Brauch zu verbinden, wonach die Hinrichtungsstätte oft als „Rosenkranz" bezeichnet wurde (z. B. in Reval). ${ }^{58}$

\footnotetext{
${ }^{56}$ vON NOTTBECK. Die alte Criminalchronik Revals (wie Anm. 26). S. 14.

${ }^{57}$ Erich KEYSER. Die Baugeschichte der Stadt Danzig, hg. v. Ernst BAHR, Köln 1972, S. 314.

${ }^{58}$ VON NOTTBECK. Die alte Criminalchronik Revals (wie Anm. 26), S. 14; JOHANSEN, vON ZUR MÜHLEN, Deutsch und Undeutsch (wie Anm. 13), S. 238.
} 